\title{
A Novel Heterozygous Missense Variant (c.667G>T;p. Gly223Cys) in USH1C That Interferes With Cadherin- Related 23 and Harmonin Interaction Causes Autosomal Dominant Nonsyndromic Hearing Loss
}

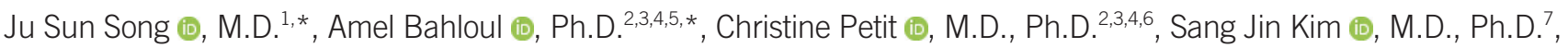

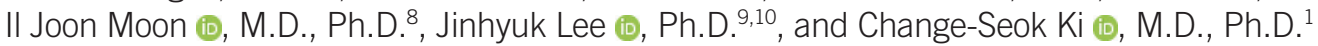

${ }^{1}$ GC Genome, Yongin, Korea; ${ }^{2}$ Unité de génétique et physiologie de l'audition, Institut Pasteur, Paris, France; ${ }^{3} U M R S$ 1120, Inserm, Paris, France; ${ }^{4}$ Sorbonne Universités, Paris, France; ${ }^{5}$ Department of Otolaryngology - Head and Neck Surgery, Stanford University, Stanford, California, USA; ${ }^{6}$ College de France and Institut Pasteur, Paris, France; ${ }^{7}$ Department of Ophthalmology, Samsung Medical Center, Sungkyunkwan University School of Medicine, Seoul, Korea; ${ }^{8}$ Department of Otorhinolaryngology-Head and Neck Surgery, Samsung Medical Center, Sungkyunkwan University School of Medicine, Seoul, Korea; ${ }^{9}$ Korean Bioinformation Center, Korea Research Institute of Bioscience and Biotechnology, Daejeon, Korea; ${ }^{10}$ Department of Bioinformatics, University of Sciences and Technology, Daejeon, Korea
\end{abstract}

Background: Pathogenic variants of USH1C, encoding a PDZ-domain-containing protein called harmonin, have been known to cause autosomal recessive syndromic or nonsyndromic hearing loss (NSHL). We identified a causative gene in a large Korean family with NSHL showing a typical pattern of autosomal dominant (AD) inheritance.

Methods: Exome sequencing was performed for five affected and three unaffected individuals in this family. Following identification of a candidate gene variant, segregation analysis and functional studies, including circular dichroism and biolayer interferometry experiments, were performed.

Results: A novel USH1C heterozygous missense variant (c.667G>T;p.Gly223Cys) was shown to segregate with the NSHL phenotype in this family. This variant affects an amino acid residue located in the highly conserved carboxylate-binding loop of the harmonin PDZ2 domain and is predicted to disturb the interaction with cadherin-related 23 (cdh23). The affinity of the variant PDZ2 domain for a biotinylated synthetic peptide containing the PDZ-binding motif of cdh23 was approximately 16-fold lower than that of the wild-type PDZ2 domain and that this inaccessibility of the binding site was caused by a conformational change in the variant PDZ2 domain.

Conclusions: A heterozygous variant of USHIC that interferes with the interaction between cdh23 and harmonin causes novel AD-NSHL.

Key Words: Harmonin, Nonsyndromic hearing loss, USH1C, Heterozygous variant
Received: June 13, 2019

Revision received: August 23, 2019

Accepted: November 26, 2019

\section{Corresponding author: Chang-Seok Ki, M.D., Ph.D. GC Genome, 107 Ihyeon-ro 30beon-gil, Giheung-gu, Yongin 16924, Korea Tel: +82-31-260-0601 Fax: +82-31-260-1595 E-mail: changski.md@gmail.com}

*These authors contributed equally to this work.

\section{(i) \$}

(c) Korean Society for Laboratory Medicine This is an Open Access article distributed under the terms of the Creative Commons Attribution Non-Commercial License (http://creativecommons.org/licenses/by-nc/4.0) which permits unrestricted non-commercial use, distribution, and reproduction in any medium, provided the original work is properly cited.

\section{INTRODUCTION}

Hearing loss $(\mathrm{HL})$ is genetically highly heterogeneous. More than 100 genes have been identified in patients with nonsyndromic $\mathrm{HL}$ (NSHL), and more than 400 genes are known to be associ- ated with some type of syndromic HL (SHL) [1]. NSHL accounts for approximately $70 \%$ of hereditary $\mathrm{HL}$, and the deafness loci (DFN) involved are named based on their mode of inheritance: DFNA (autosomal dominant, AD), DFNB (autosomal recessive, $A R$ ), and DFNX (X-linked). 
Most forms of hereditary HL caused by pathogenic variants (PVs) of a specific gene show only one inheritance pattern; however, a few can have either AD or AR mode of inheritance such as those associated with PVs of GJB2, GJB6, MYO7A, and TECTA [1]. USH1C, encoding harmonin, is known to be related to DFNB18, AR retinitis pigmentosa (RP) with late-onset $\mathrm{HL}$, and sector RP and $\mathrm{HL}$, as well as Usher syndrome type 1 [2-6]. All these phenotypes are inherited in the AR mode; an AD inheritance pattern has yet to be reported.

We identified a novel USH1C variant in a large Korean family with NSHL. Interestingly, this family showed a typical pattern of $A D$ inheritance and the USHIC variant segregated perfectly with NSHL. Functional analysis of the USH1C variant revealed impaired binding between cdh23 and the PDZ2 domain of harmonin and suggested a novel pathogenic mechanism, in which a USH1C variant caused AD-NSHL.

\section{MATERIALS AND METHODS}

Individuals and clinical evaluation

In 2016, a three-generation, 48-member Korean family presenting with AD-NSHL was identified at Samsung Medical Center, Seoul, Korea (Fig. 1A). The proband (III-21) underwent complete ophthalmic examinations, including visual-acuity measurement, slit lamp biomicroscopic examination, fundus examination, color vision test, full-field electroretinogram, color fundus photography, fundus autofluorescence, and spectral domain optical coherence tomography. Audiologic evaluations included pure tone audiometry with air and bone conduction, speech audiometry, impedance audiometry with tympanometry and stapedial reflex, otoacoustic emission, and auditory brainstem response. Vestibular function of the proband was evaluated by caloric test, vestibular evoked myogenic potential, rotatory chair test, and sensory organization test. This study was approved by the Institutional Review Board of Samsung Medical Center, and written informed consent was obtained from all individuals who participated in this study.

\section{Clinical findings}

Detailed family history revealed the pedigree of the three-generation, 48-member Korean family with NSHL showing a pattern of $A D$ inheritance (Fig. 1A). For available family members, onset of $\mathrm{HL}$ occurred during adolescence in all affected individuals except one (II-13) with prelingual HL and another one (III-16) with later onset in the mid-twenties. Other related clinical manifestations suggesting SHL were not present in any of the affected individuals (Table 1).

Comprehensive ophthalmologic examinations, including fundus photography and electroretinogram of the proband (III-21), showed normal findings (Fig. 1B and $1 \mathrm{C}$ ). Pure-tone audiometry demonstrated bilateral sensorineural $\mathrm{HL}$ with a pure-tone average $(0.5,1,2$, and $4 \mathrm{kHz})$ of $53 \mathrm{~dB}$ and $56 \mathrm{~dB}$ in the right and left ear, respectively (Fig. 1D). The word recognition scores of the right and left ears, presented at the most comfortable level, were $96 \%$ and $94 \%$, respectively. Tympanometry indicated a normal type A curve for the tympanogram, and the stapedius reflex (acoustic) was present at all sound frequencies. Distortion product and transient evoked otoacoustic emissions were absent on both sides. The thresholds obtained by auditory brainstem response recordings were $60 \mathrm{~dB}$ for both ears. The caloric test, vestibular evoked myogenic potential, rotatory chair test, and sensory organization test showed normal or unremarkable findings (Fig. 1E).

\section{Whole exome sequencing (WES)}

To identify a causative gene in this family, we performed WES using genomic DNA extracted from peripheral blood leukocytes. In brief, $3 \mathrm{~mL}$ of whole blood was drawn from each individual, and $200 \mu \mathrm{L}$ of fresh whole blood was used to extract genomic DNA using MagNA Pure 96 Instrument (Roche Diagnostics, Basel, Switzerland). Five affected (II:4, II:6, III:16, III:20, and III:21) and three unaffected individuals (III:6, III:12, and III:17) were selected for WES considering the distance of relatedness [7]. Library preparation was carried out using SureSelect Human All Exon V5 (Agilent Technologies, Santa Clara, CA, USA), and sequencing was performed using NextSeq500 (Illumina Inc., San Diego, CA, USA), generating $2 \times 150$ bp paired-end reads. Alignment of sequence reads, indexing of the reference genome (hg19), and variant calling were conducted using the GATK Best Practice pipeline (https://software.broadinstitute.org/ gatk/best-practices/).

\section{Identification of causative gene}

Considering the AD pattern of inheritance, we filtered heterozygous variants $(0.4<$ variant allele frequency $[V A F]<0.6)$ that were shared by the five affected individuals and absent $(\mathrm{VAF}=0)$ in the three unaffected individuals as candidates. The variants were restricted to those with a population minor allele frequency $<1 \%$ in the 1000 Genomes Project database [8], the Exome Aggregation Consortium database (ExAC; http://exac.broadinstitute.org/), or the Korean Reference Genome Database (KRGDB; http://152.99.75.168/KRGDB/). The variant identified in the seg- 
A

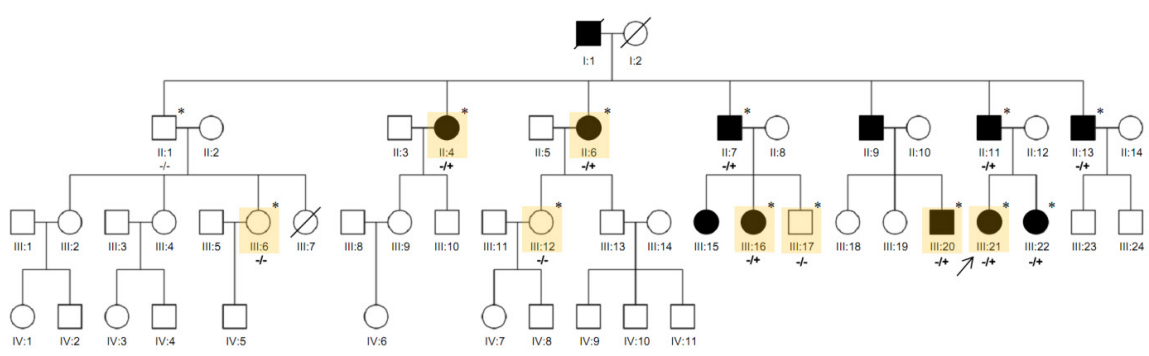

B

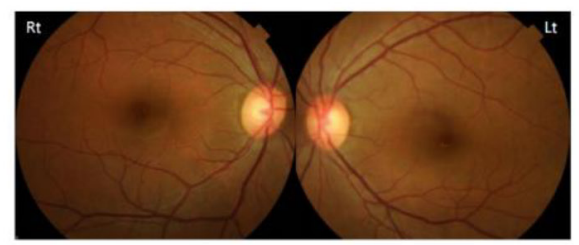

C

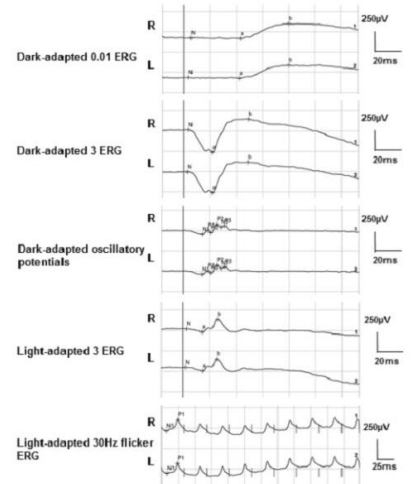

D

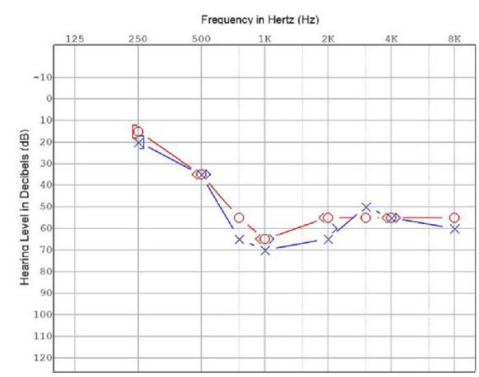

E

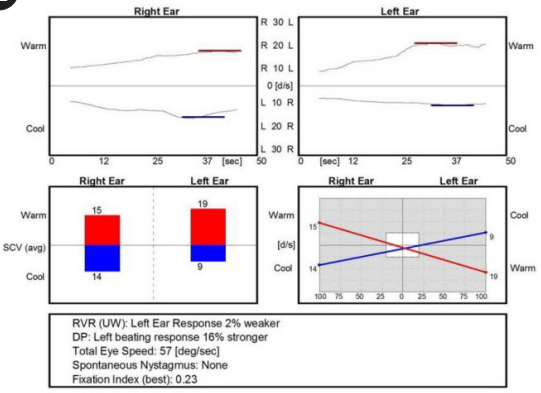

F
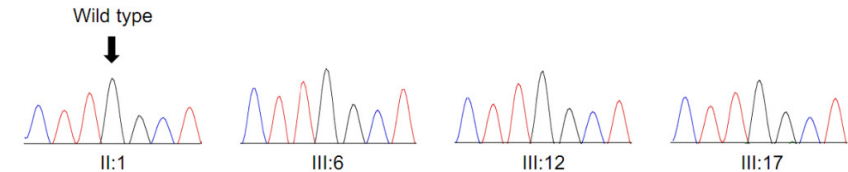

c.667G>T (p.Gly223Cys)
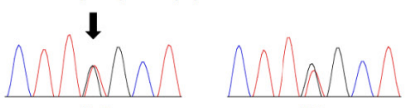

11.6
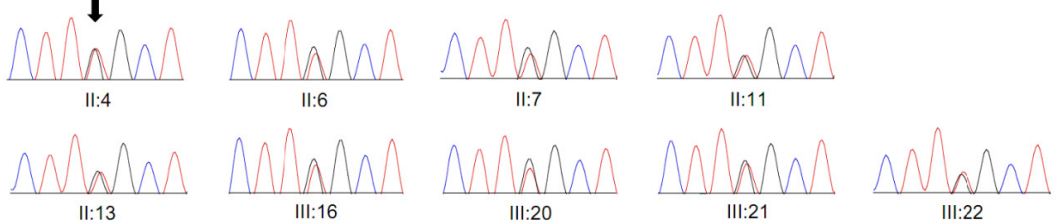

Fig. 1. Clinical and genetic findings in a Korean family with NSHL. (A) Pedigree of a large Korean family with HL; the proband is indicated with an arrow. Individuals who participated in the molecular genetic testing are marked with an asterisk on the right. Whole exome sequencing was performed for eight individuals (shaded in yellow). The presence (+) or absence (-) of the USH1C c.667G > T variant is indicated underneath each individual. (B) Fundus autofluorescence photographs and (C) full-field electroretinogram showing normal findings. (D) Pure tone audiometry result showing moderate to moderately severe $\mathrm{HL}$, with an average of 53 and $56 \mathrm{~dB}$ for the right and left ears, respectively. (E) Caloric test result showing normal vestibular function bilaterally. (F) Sequencing chromatograms of the c.667G $>$ T variant of USH1C in individuals who participated in the molecular genetic testing. Nomenclature is based on RefSeq NM_005709.3, with nucleotide number +1 designating the $\mathrm{A}$ of the start codon ATG.

Abbreviations: HL, Hearing loss; NSHL, nonsyndromic hearing loss; Lt, left; Rt, right. 
Table 1. Clinical findings of family members with NSHL

\begin{tabular}{|c|c|c|c|c|c|}
\hline Individual* & Gender & Age (yr) & Life stage at onset (yr) & $\mathrm{HL}$ & Verbal function \\
\hline$\|-1$ & Male & 74 & - & No & Normal \\
\hline |I-4 & Female & 73 & Adolescence & Use of hearing aids & Normal \\
\hline II-6 & Female & 67 & Adolescence & Use of hearing aids & Normal \\
\hline II-7 & Male & 64 & Adolescence & Use of hearing aids & Normal \\
\hline ||-11 & Male & 58 & Adolescence & Pure-tone average: $85 \mathrm{~dB}$ in right ear and $80 \mathrm{~dB}$ in left ear & Normal \\
\hline$\|-13$ & Male & 55 & Birth & Use of hearing aids & Lalopathy \\
\hline III-6 & Female & 43 & - & No & Normal \\
\hline ||I-12 & Female & 40 & - & No & Normal \\
\hline |II-16 & Female & 31 & Mid-twenties & Use of hearing aids & Normal \\
\hline ||l-17 & Male & 36 & - & № & Normal \\
\hline III-20 & Male & 31 & Adolescence & Diagnosed by pure-tone audiogram in army (data not available) & Normal \\
\hline III-21 $1^{\dagger}$ & Female & 32 & Adolescence & Pure-tone average: $53 \mathrm{~dB}$ in right ear and $56 \mathrm{~dB}$ in left ear & Normal \\
\hline ||I-22 & Female & 24 & Adolescence & Pure-tone average: $43 \mathrm{~dB}$ in right ear and $27 \mathrm{~dB}$ in left ear & Normal \\
\hline
\end{tabular}

${ }^{*}$ None had tinnitus, vertigo, and other relevant clinical manifestations; ${ }^{\dagger}$ proband.

Abbreviation: NSHL, nonsyndromic hearing loss; HL, hearing loss.

Table 2. Segregation analysis using exome sequencing data and statistics

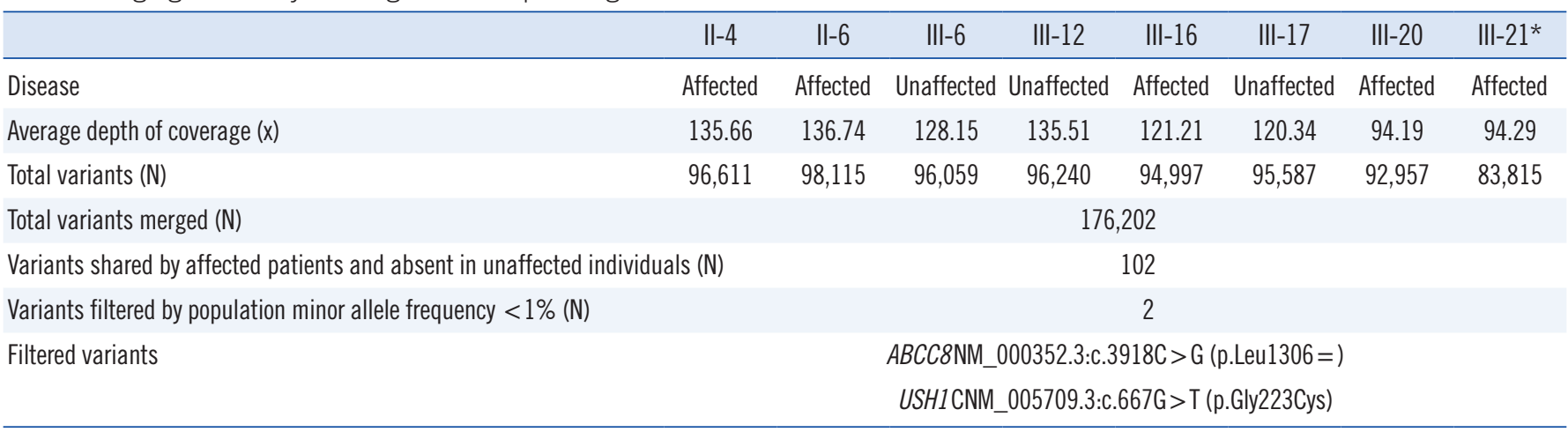

*proband.

regation analysis was confirmed by Sanger sequencing using primers designed by the authors (available on request) in five individuals who underwent WES (II:4, II:6, III:16, III:20, III:21) as well as four additional family members with NSHL (II:7, II:11, II:13, III:22).

\section{Functional studies}

Following identification, the candidate variant of USH1C was introduced into a harmonin cDNA fragment encoding the full-length harmonin- a isoform and PDZ2 domain (amino acids 197 to 208; GenBank accession No. AF228924) and cloned into the pGST//2 vector. Recombinant proteins were expressed purified as described previously [9]. The differences in the measured affinity between the wild-type PDZ2 and the PDZ2 variant with cdh23 could be attributed to the inaccessibility of the binding site in the variant PDZ2 and this could result either from the presence of a bulky and polar cysteine residue instead of glycine at position 223 or from a conformational change of the PDZ2 domain induced by the variant. We therefore used circular dichroism spectra to compare the global secondary structure of the wild-type (WT) and variant PDZ2 domains. Circular dichroism experiments on the PDZ2 domains were performed using an Aviv 215 spectropolarimeter (Aviv Biomedical, Inc., Toms River, NJ, USA). Far-UV (195-260 nm) spectra were recorded at $20^{\circ} \mathrm{C}$ in $50 \mathrm{mM}$ Tris [pH 8], $200 \mathrm{mM} \mathrm{KCl}$, and $0.5 \mathrm{mM}$ Tris (2-carboxyethyl) phosphine (TCEP). The wavelength range used was 195$230 \mathrm{~nm}$, with a band width of $5.0 \mathrm{~nm}$.

To investigate the potential dominant negative effect of the harmonin variant, we examined two mutually nonexclusive possibilities. One is sequestration of partners due to the variant, and the 
other is the formation of hetero-oligomers between the WT and variant protein. Both the WT and variant full-length harmonin used in this study were individually purified and subsequently subject to size-exclusion chromatography (SEC) [9] to compare the elution volume of the analytes in solution $(50 \mathrm{mM}$ Tris $[\mathrm{pH}$ 8], $200 \mathrm{mM} \mathrm{NaCl}$, and $0.1 \mathrm{mM}$ TCEP) on the basis of their respective sizes.

Biolayer interferometry (BLI) experiments were performed using an Octet Red 384 instrument (Pall ForteBio, Fremont, CA,
USA) [10]. Since the Octet instrument allows performing binding assays at $4^{\circ} \mathrm{C}$ above the ambient temperature, the room temperature was maintained around $20^{\circ} \mathrm{C}$ to perform the binding studies at $25^{\circ} \mathrm{C}$. Streptavidin biosensors (Pall ForteBio) were loaded at room temperature with a biotin-tagged synthetic peptide comprising 12 amino acid residues, including the C-terminal PDZbinding motif (PBM) sequence of cdh23. Reference sensors were loaded with biocytin. Both series of biosensors were then incubated in parallel for 2 min at $25^{\circ} \mathrm{C}$ with proteins at different con-

(A)

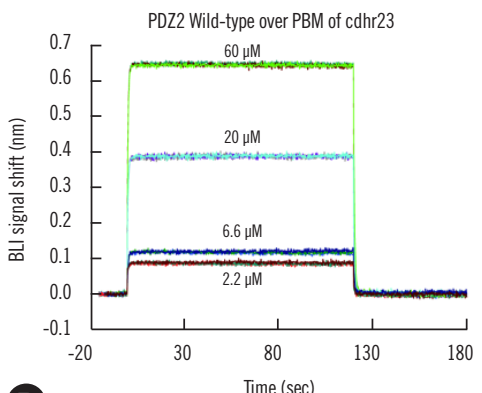

B

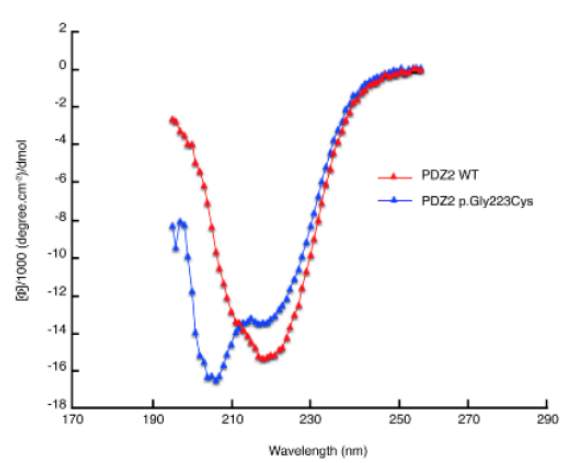

(D)
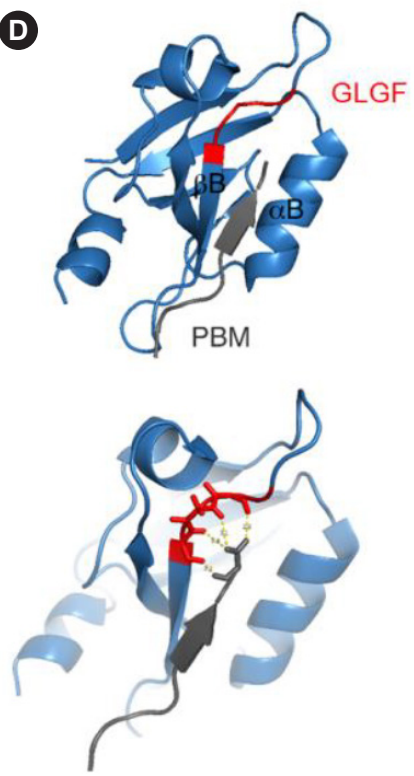

PDZ2 G223C over PBM of cdhr23
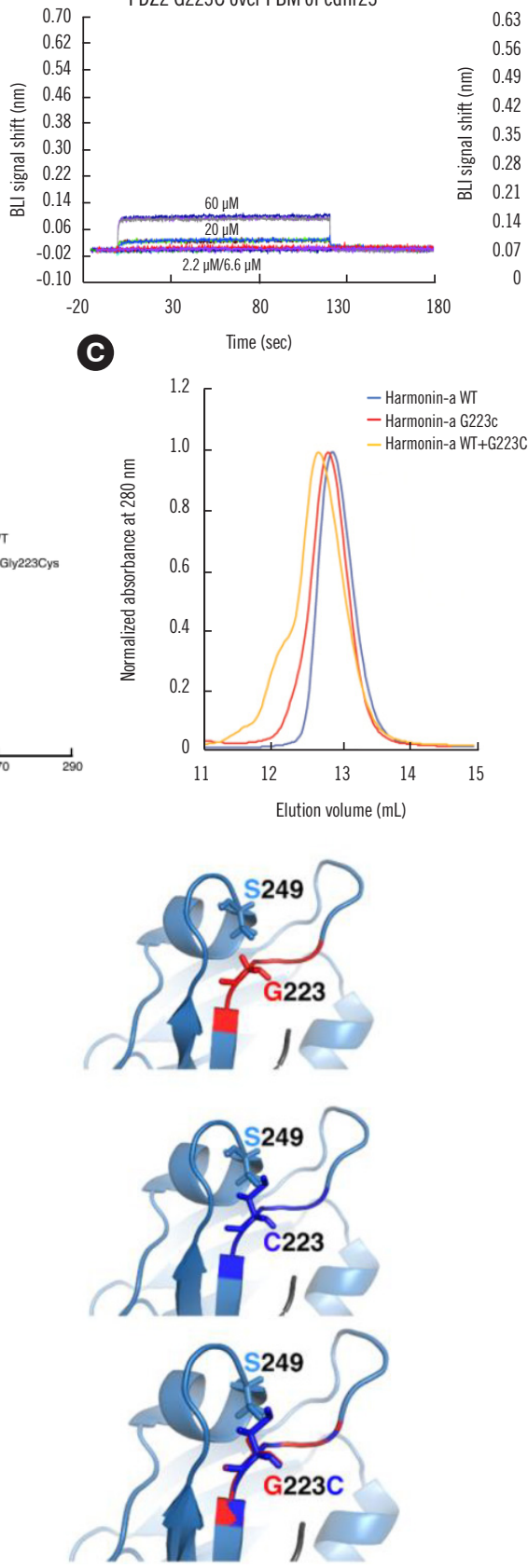

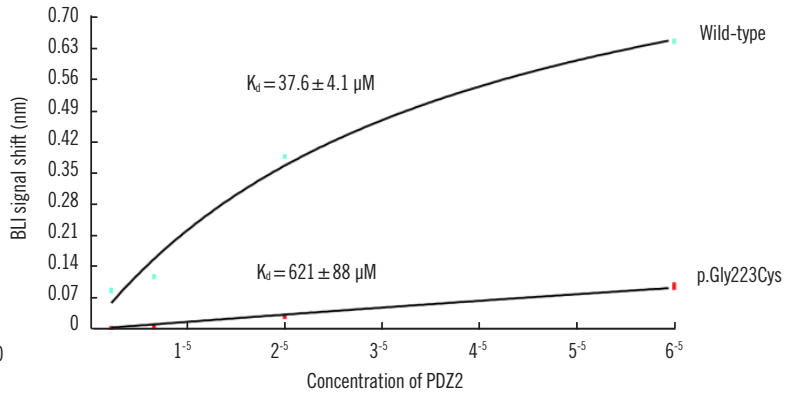

Fig. 2. Functional analysis and protein modeling of harmonin. (A) The affinity of the WT PDZ2 $\left(K_{d}=37.6 \pm\right.$ $4.1 \mu \mathrm{M}$ ) was approximately 16 -fold higher than that of PDZ2 carrying the p.Gly223Cys variant $\left(K_{d}=621 \pm 88\right.$ $\mu \mathrm{M})$. (B) Circular dichroism spectra for PDZ2 WT and the PDZ2 p.Gly223Cys variant demonstrated the global secondary structure of the WT and variant PDZ2 domain and showed that the p.Gly223Cys variant causes a conformational change. (C) The normalized elution profile of the full-length p.Gly223Cys variant protein showed a left-shifted peak compared with WT full-length harmonin, and a mixture of the full-length p.Gly223Cys variant and WT harmonin revealed a further left-shifted peak. (D) The structure of the PDZ2 domain of harmonin in a complex with the PBM of cdh23 and conformational analysis of the p.Gly223Cys variant demonstrated the interaction between the PDZ2 domain of harmonin and the PDZ-binding motif of cdh23 through the GLGF motif, a highly conserved carboxylate-binding loop in a groove between the $\alpha \mathrm{B}$ and $\beta \mathrm{B}$ structural elements (left). The third glycine residue in the loop region is fully conserved and adopts a left-handed $\alpha$-helical conformation. Replacement of the glycine residue with cysteine leads to a conformational change with a structural modification (right). Abbreviations: WT, wild-type; SEC, size-exclusion chromatography; PBM, PDZ-binding motif. 
centrations ranging from 2.2 to $60 \mu \mathrm{M}$ in $50 \mathrm{mM}$ Tris ( $\mathrm{pH}$ 8), $200 \mathrm{mM} \mathrm{KCl}$, and $0.5 \mathrm{mM}$ TCEP. Signals measured on the reference sensors were subtracted from those measured on the peptide sensors. The affinities of the WT and variant PDZ2 were determined by analyzing the concentration-dependence of steadystate BLI signals.

\section{RESULTS}

\section{Identification of USH1C variant}

The process of segregation analysis using WES data is presented in Table 2. Two variants were filtered. One was a novel heterozygous missense variant (NM_005709.3:c.667G > T;p.Gly223Cys) of USH1C, which is a known gene related to HL. Sanger sequencing confirmed co-segregation of the USHIC variant with the NSHL phenotype in this family. The variant was identified in all affected participating family members, i.e., the five affected individuals (II:4, II:6, III:16, III:20, III:21) and four affected relatives (II:7, II:11, II:13, III:22); however, it was not present in the three unaffected family members (III:6, III:12, III:17) and in an additional unaffected individual analyzed by Sanger sequencing (II:1; Fig. 1F). All other variants of USH1C and genes known to be associated with $\mathrm{HL}$ were either classified as benign or likely benign or did not segregate with the NSHL phenotype in this family. The other variant was an $A B C C 8$ synonymous variant (NM_000352.3:c.3918C > G;p.Leu1306=), which is listed in the dbSNP (rs372186045; https://www.ncbi.nlm.nih.gov/snp/ rs372186045/), with a frequency of $0.26 \%$ in the East Asian population. Considering that the distance between the USH1C and $A B C C 8$ variants is only $129 \mathrm{~kb}$ on chromosome 11 , the synonymous $A B C C 8$ variant may be in linkage disequilibrium with the USHIC variant.

\section{Functional effect of the USH1C variant}

The affinity of the WT PDZ2 for a biotinylated synthetic peptide containing the PBM of cdh23 (biotin-TtdsVIMESPLEITEL) was approximately 16 -fold higher $\left(K_{d}=37.6 \pm 4.1 \mu \mathrm{M}\right)$ than that of the variant PDZ2 carrying the missense USH1C variant $\left(\mathrm{K}_{\mathrm{d}}=\right.$ $621 \pm 88 \mu \mathrm{M}$; Fig. 2A). The different shapes of the curves obtained in the circular dichroism experiments indicated that the variant causes a conformational change in the PDZ2 domain (Fig. 2B).

In SEC experiments, WT full-length harmonin showed a single elution peak in the chromatogram, corresponding to the monomeric state of the protein, whereas the variant full-length harmonin showed a slightly leftward shift of the elution peak (Fig. 2C).
When both WT and variant full-length harmonins were mixed and subjected to SEC, a more pronounced leftward shift of the peak was observed, accompanied by the appearance of another peak further leftward, indicating the elution of a higher molecular weight species. The difference in the elution volume, reflected in the differentially positioned peaks, potentially indicates the formation of a hetero-dimer between the WT and variant harmonins.

\section{DISCUSSION}

We report a large Korean family with a heterozygous USH1C missense variant and provide evidence suggesting a role for this gene in AD-NSHL. Stereocilia in the inner ear hair cell are coupled to one another by a variety of links, among which tip links, composed of cdh23 and cdh15, are the most crucial for mechano-electrical transduction [11]. Harmonin is found at the upper attachment of the tip link and forms a stable anchorage structure complex with cdh23 at the tip link of stereocilia via multivalent interactions [12]. Harmonin in retinal photoreceptors is localized at synaptic terminals and may play essential roles in the structural and functional unity of this synaptic junction; however, its function remains unknown [13, 14].

The PDZ domains in harmonin mediate the interaction between cdh23 and harmonin. These domains comprise 80-90 amino acid residues that contain six $\beta$-strands and two $\alpha$-helices and have a single binding site in a groove between the $\alpha \mathrm{B}$ and $\beta B$ structural elements, with a highly conserved carboxylate-binding loop (R/K-XXX-G- $\Phi-G-\Phi$ motif, where $X$ is any amino acid residue and $\Phi$ is a hydrophobic residue) located before the $\beta B$ strand (Fig. 2D) [15]. The p.Gly223Cys variant of harmonin affects the third residue of the GLGC motif in the carboxylate-binding loop of the PDZ2 domain, and it is predicted that this amino acid substitution disrupts the interaction between the PBM of cdh23 and the PDZ2 domain of harmonin [16]. In addition, the third glycine residue in the loop is fully conserved and adopts a left-handed $\alpha$-helical conformation in all structures reported to date, and this residue may be important for determining the PDZ fold [17]. Therefore, the p.Gly223Cys variant in harmonin is likely to exert significant functional consequences by reducing the accessibility of the PDZ binding site to the target and inducing a structural modification.

The USH1C c.667G > T (p.Gly223Cys) variant was shown to be absent in 1,722 individuals in the KRGDB and 60,706 individuals in the ExAC. When this variant was classified according to the Sequence Interpretation Guidelines of the American College of Medical Genetics and Genomics and the Association for 
Molecular Pathology [7], it was interpreted as a likely PV (LPV) based on the following evidence: absent in population database (PM2), multiple lines of computational evidence supporting a deleterious effect on the gene (PP3), and co-segregation with disease in multiple affected family members (from supporting evidence PP1 to strong evidence with a large extent of segregation). If a well-established functional study showing a deleterious effect (PS3) could be conducted based on the present data, the variant classification might change from LPV to PV.

This study shows that a single PV in USH1C can cause disease in NSHL patients. The USHIC PVs reported thus far are mostly null variants (including nonsense, frameshift, and splicing mutations) on both gene copies with some exceptions: a compound variant heterozygous for a null and a missense mutation, a homozygous missense change variant, and a homozygous "leaky" splice-site mutation variant. AD transmission related with $\mathrm{HL}$ has yet to be described to the best of our knowledge [5]. Therefore, conventional genetic diagnostic approaches based on the well-known inheritance pattern of each gene in NSHL may fail to identify the real causative variant in a minor portion of NSHL patients.

\section{ACKNOWLEDGEMENTS}

We thank the patients and family members for giving us an opportunity to identify the genetic cause of $\mathrm{HL}$ in this family. We are particularly grateful to Patrick England for his help in affinity measurement.

\section{AUTHOR CONTRIBUTIONS}

JSS and CSK contributed to the conception and design of the study. SJK and IJM were involved in clinical evaluation of all patients and their family members. JSS conducted sample acquisition, collected clinical information, and analyzed WES data. $A B$ and $C P$ performed functional analysis and $J L$ analyzed the protein structure. JSS and AB wrote the manuscript, which was revised critically by all authors. CSK supervised this study and is the guarantor of this article. All authors read and approved the final manuscript.

\section{CONFLICTS OF INTEREST}

No potential conflicts of interest relevant to this paper were reported.

\section{RESEARCH FUNDING}

This study was supported by the Intramural Research Funds from Samsung Medical Center.

\section{ORCID}

Ju Sun Song

https://orcid.org/0000-0003-4607-872X

Amel Bahloul https://orcid.org/0000-0001-7042-4616

Christine Petit https://orcid.org/0000-0002-9069-002X

Sang Jin Kim https://orcid.org/0000-0002-1502-3155

II Joon Moon https://orcid.org/0000-0002-3613-0734

Jinhyuk Lee https://orcid.org/0000-0002-7437-1846

Change-Seok Ki https://orcid.org/0000-0001-7679-8731

\section{REFERENCES}

1. Shearer AE, Hildebrand MS, Smith RJH. Hereditary hearing loss and deafness overview. In: Adam MP, Ardinger HH, Pagon RA, et al., eds. GeneReviews [Internet]. Seattle, WA, 1993. https://www.ncbi.nlm.nih. gov/books/NBK1434/ (Updated on July 2017).

2. Blaydon DC, Mueller RF, Hutchin TP, Leroy BP, Bhattacharya SS, Bird $\mathrm{AC}$, et al. The contribution of USHIC mutations to syndromic and nonsyndromic deafness in the UK. Clin Genet 2003;63:303-7.

3. Ahmed ZM, Smith TN, Riazuddin S, Makishima T, Ghosh M, Bokhari S, et al. Nonsyndromic recessive deafness DFNB18 and Usher syndrome type IC are allelic mutations of USHIC. Hum Genet 2002;110:527-31.

4. Ouyang XM, Xia XJ, Verpy E, Du LL, Pandya A, Petit C, et al. Mutations in the alternatively spliced exons of USH1C cause non-syndromic recessive deafness. Hum Genet 2002;111:26-30.

5. Khateb S, Zelinger L, Ben-Yosef T, Merin S, Crystal-Shalit O, Gross M, et al. Exome sequencing identifies a founder frameshift mutation in an alternative exon of USH1C as the cause of autosomal recessive retinitis pigmentosa with late-onset hearing loss. PLoS One 2012;7:e51566.

6. Saihan Z, Stabej Ple Q, Robson AG, Rangesh N, Holder GE, Moore AT, et al. Mutations in the USH1C gene associated with sector retinitis pigmentosa and hearing loss. Retina 2011:31:1708-16.

7. Richards S, Aziz N, Bale S, Bick D, Das S, Gastier-Foster J, et al. Standards and guidelines for the interpretation of sequence variants: a joint consensus recommendation of the American College of Medical Genetics and Genomics and the Association for Molecular Pathology. Genet Med 2015;17:405-24

8. 1000 Genomes Project Consortium, Auton A, Brooks LD, Durbin RM, Garrison EP, Kang HM, et al. A global reference for human genetic variation. Nature 2015;526:68-74.

9. Bahloul A, Pepermans E, Raynal B, Wolff N, Cordier F, England P, et al. Conformational switch of harmonin, a submembrane scaffold protein of the hair cell mechanoelectrical transduction machinery. FEBS Lett 2017; 591:2299-2310.

10. Kamat V, Rafique A. Designing binding kinetic assay on the bio-layer interferometry (BLI) biosensor to characterize antibody-antigen interactions. Anal Biochem 2017;536:16-31.

11. Ahmed ZM, Frolenkov GI, Riazuddin S. Usher proteins in inner ear structure and function. Physiol Genomics 2013;45:987-9. 
Song JS, et al.

A novel USH1C heterozygous missense variant

ANNALS OF

LABORATORY MEDICINE

12. Wu L, Pan L, Zhang C, Zhang M. Large protein assemblies formed by multivalent interactions between cadherin23 and harmonin suggest a stable anchorage structure at the tip link of stereocilia. J Biol Chem 2012; 287:33460-71.

13. Cosgrove D and Zallocchi M. Usher protein functions in hair cells and photoreceptors. Int J Biochem Cell Biol 2014;46:80-9.

14. Reiners $\mathrm{J}$ and Wolfrum $U$. Molecular analysis of the supramolecular usher protein complex in the retina. Harmonin as the key protein of the Usher syndrome. Adv Exp Med Biol 2006;572:349-53.
15. Kalyoncu S, Keskin O, Gursoy A. Interaction prediction and classification of PDZ domains. BMC Bioinformatics 2010;11:357.

16. Siemens J, Kazmierczak P, Reynolds A, Sticker M, Littlewood-Evans A, Müller $\mathrm{U}$. The Usher syndrome proteins cadherin 23 and harmonin form a complex by means of PDZ-domain interactions. Proc Natl Acad Sci U S A 2002;99:14946-51.

17. Lee HJ and Zheng JJ. PDZ domains and their binding partners: structure, specificity, and modification. Cell Commun Signal 2010;8:8. 